\title{
Editorial
}

\section{Obesity - what role now for nutritional science?}

For the last three or four decades obesity has been widely considered the major nutritional disorder in the Western world, and over the past 20 years its incidence has increased dramatically. In the UK, for example, in the early $1980 \mathrm{~s}$ just $6 \%$ of adult men and $8 \%$ of adult women were classified as obese $\left(\mathrm{BMI}>30 \mathrm{~kg} / \mathrm{m}^{2}\right)$, while the figure is now approximately $22 \%$ of all UK adults. Similarly, there has been a recent rapid increase in obesity in children. Being obese is associated with a reduction in life expectancy of some 8 years, and there is an increased incidence of several major diseases, particularly diabetes, coronary heart disease and certain cancers.

The point has been reached where obesity is now cited as being as important as smoking in the causation of cancer. In the case of diabetes, again using the UK as an example, a very recent report has indicated that nearly 1.8 million people are currently diagnosed as diabetic, representing $3 \%$ of the total population (Diabetes UK, 2004). This figure is expected to rise to 3 million, or $5 \%$ of the population, by the year 2010 - and most of this increase is a direct consequence of the surge in obesity. Worldwide, there are currently estimated to be 150 million diabetics, with a projection of 300 million by 2025 .

The personal and social costs of obesity and its associated disorders can be considerable. There are also substantial economic costs, not least in relation to treatment. Treating diabetes alone in the UK now consumes $5 \%$ of the expenditure of the national health-care system and the obesity-related rise in the number of diabetics by 2010 is likely to push this proportion to $10 \%$ (equivalent to $€ 15$ billion per annum). The rising social and economic costs have led to engagement in the issue by politicians and governments. Indeed, this is the latest stage in the development of obesity as very much a multidisciplinary field; it has increasingly encompassed public health, social, cultural, behavioural and political dimensions, in addition to the strictly biological. Dietary advice (on an individual or group level) and behavioural modification have, of course, long been part of the scene in obesity, including the distinctive contribution of commercial organisations.

The emerging political dimension is symbolised in the UK by a recent enquiry on obesity by the House of Commons (Parliamentary) Health Committee (House of Commons Health Committee, 2004). While in some respects the fact that obesity is now firmly on the political agenda can be welcomed, it is appropriate to inject some caution. There is a risk that obesity could become viewed as an issue whose remedy is essentially through government action, and the subject of ministerial targets, thereby minimising the responsibilities of the individual in life-style decisions in relation to diet and physical activity. Indeed, there must be a concern that, in some societies, government intervention - no matter how well intentioned and evidence-based - will prove to be counter-productive unless conducted with considerable subtlety.

In view of the multidisciplinary nature of obesity, and the emerging political involvement, it is appropriate to consider the particular contribution that nutritional science and other areas of biology make to a field which until recently was primarily their own. The issue has added resonance given that obesity is the result of energy intake exceeding expenditure, and as such is not perceived as requiring 'high science'. The issue is best addressed by defining the landscape covered by the 'biology of obesity'. In my view this landscape encompasses the following areas, some of which are at the core of nutritional science: (i) the fundamental mechanisms of energy balance and its regulation (appetite, energy expenditure, endocrine factors, neurobiology, genes); (ii) the biological basis of the development of obesity (again genes, appetite, energy expenditure); (iii) adipose tissue function (lipid metabolism and lipidomics, adipokines); (iv) the biology of the obese state (nutrient partitioning, substrate flux, physiological adaptations); (v) the pathological consequences of obesity (mechanistic basis of the development of the associated disorders); and (vii) the physiological basis of treatment strategies, both established and novel (whether nutritional, behavioural or pharmacological).

In addition to classical nutritional and physiological approaches and techniques, the biology of obesity requires the application of transcriptomics, proteomics and metabolomics. Genomic approaches are well entrenched, with the search for single gene mutations and gene polymorphisms which might predispose to increased body fat, as well as the use of transgenics to identify key genes and regulatory systems in energy balance.

A novel approach has recently been reported using genome-wide RNAi analysis of the fat regulatory genes in the worm Caenorhabditis elegans (Ashrafi et al. 2003). The estimated 16737 worm genes were systematically suppressed using RNAi, and 417 of these inactivations were found to result in alterations in body fat. Of these, 305 reduced fat while 112 led to increases (Ashrafi et al. 2003). Although some of the identified genes were predictable from mammalian studies, others were not. For example, inactivation of GAPDH or a $\mathrm{K}$ channel resulted in a reduction in body fat; in contrast, inactivation of the glutamate receptor or a chemoreceptor led to increased fat.

The application of genome-wide RNAi analysis in worms has the real potential to identify new systems which impact on body fat regulation in mammals and to provide novel candidate genes in the search for the underlying genetic basis of obesity 
in humans. More broadly, it is also a potent example of what radically different perspectives can contribute to nutritional science.

Paul Trayhurn

Editor-in-Chief School of Clinical Sciences University of Liverpool, Liverpool L69 3GA UK p.trayhurn@liverpool.ac.uk

\section{References}

Ashrafi K, Chang FY, Watts JL, Fraser AG, Kamath RS, Ahringer J \& Ruvkun G (2003) Genome-wide RNAi analysis of Caenorhabditis elegans fat regulatory genes. Nature 421, 268-272.

Diabetes UK (2004) Diabetes in the UK 2004. London: Diabetes UK.

House of Commons Health Committee (2004) Obesity - Third Report of Session 2003-04. London: The Stationery Office. 